\title{
O Acometimento Isquêmico Obstrutivo na Cardiomiopatia Hipertrófica do Tipo Septal Assimétrico com Envolvimento da Artéria Descendente Anterior
}

\author{
Francisco Manes Albanesi F, Marcia Bueno Castier, Tatiana Tavares da Silva, \\ Silvia Helena Cardoso Boghossian, Paulo Ginefra
}

Rio de Janeiro, RJ

Objetivo - Avaliar o papel da doença arterial coronária (DAC) com comprometimento da artéria descendente anterior (ADA) na cardiomiopatia hipertrófica (CMH) e sua repercussão na evolução, visto ser controverso o significado da necrose e fibrose do septo interventricular (SIV) nesta cardiomiopatia.

Métodos - Entre 158 pacientes com CMH, selecionamos 6(3,79\%) com CMH e DAC com lesão obrigatória de ADA, sendo 4 homens, entre 52 e $70(x=65,16)$ nos, 4 com a forma obstrutiva da CMH. O tempo de diagnóstico da CMH foi de 78 a $182(x=141)$ meses e da DAC de 1 dia a 106 $(x=42)$ meses. Os pacientes foram acompanhados com avaliações clínicas e exames complementares periódicos.

Resultados - A forma de apresentação da DAC foi em 5 com angina instável e um com infarto do miocárdio. A ADA estava comprometida entre 60 a $100 \%$, sendo em um lesão única e nos 5 restantes com lesão em 2 ou mais vasos. $\mathrm{Na}$ evolução, 3 foram submetidos a revascularização miocárdica (RM), um associada a miomectomia septal, um a angioplastia e 2 somente a tratamento clínico. No período de observação de 76 a 124 meses após o diagnóstico da DAC, ocorreu um óbito. No fim do estudo observamos redução nos valores médios do SIV de 1,53 para $1,40 \mathrm{~cm}$, gradiente de pressão entre o corpo e a via de saída do ventrículo esquerdo (VE) de 56 para $15,75 \mathrm{mmHg}$, com discreto aumento no diâmetro diastólico do VE de 4,55 para 4,85cm e do diâmetro sistólico de 2,83 para $3,13 \mathrm{~cm}$, sem alterar a dimensão do átrio esquerdo $(4,13 \mathrm{~cm})$.

Conclusão - A DAC da ADA é bem tolerada na $\mathrm{CMH}$ septal assimétrica, participando do processo fibrótico septal e melhorando o desempenho cardíaco, não representando problema adverso na evolução da $\mathrm{CMH}$.

Palavras-chaves: cardiomiopatia hipertrófica, cardiopatia isquêmica obstrutiva, lesão da artéria descendente anterior

\section{Obstructive Ischemic Involvement of the Left Anterior Descending Coronary Artery in Asymmetrical Septal Form of Hypertrophic Cardiomyopathy}

Purpose - The significance of necrosis and fibrosis of the interventricular septum in hypethrophic cardiomyopathy (HCM) is controversial. The purpose of this study was then to evaluate the clinical impact of left anterior descending artery (LAD) disease in HCM.

Methods - Among 158 patients presenting with HCM, 6 (3.79\%) had LAD disease. Mean age was 65.16 years (52 to 70), 4 were men and 4 had the obstructive form of HCM. All patients were submitted to complete clinical and laboratory evaluation. Mean time of the diagnosis was 141 months (ranging from 78 to 182) for HCM and 42 months (ranging from one day to 106 months) for LAD disease.

Results - Five patients had unstable angina and one had myocardial infarction. LAD disease (60 to $100 \%$ coronary narrowing) was present in all patients; one patient had single vessel disease and 5 multivessel disease. During follow-up, 3 patients had coronary artery bypass grafting $(C A B G)$, one with associated septal myectomy; one was submitted to coronary angioplasty and 2 were submitted to medical treatment. During a follow-up period ranging from 76 to 124 months after LAD disease diagnosis, one patient died. At the end of the study, a reduction of the thickness of the interventricular septum from 1.53 to $1.40 \mathrm{~cm}$ was observed and left ventricular outflow pressure gradient decreased from 56 to $16 \mathrm{~mm} \mathrm{Hg}$. Left ventricular diastolic diameterincreased from 4.55 to $4.85 \mathrm{~cm}$ and systolic diameterfrom 2.83 to $3.13 \mathrm{~cm}$. Left atrium diameterwas unchanged.

Conclusion - LAD disease is well tolerated in the asymmetrical form of HCM and may contribute to septal fibrosis, improving cardiac function. It does not represent an adverse factor in the evolution of HCM.

Key-words: hypertrophic cardiomyopathy, atherosclerotic coronary artery disease, left anterior descending coronary artery lesion

Arq Bras Cardiol, volume 69 (n 5), 309-315, 1997

Universidade do Estado do Rio de Janeiro - UERJ

Correspondência: Francisco Manes Albanesi F $F^{\circ}$ Av. 28 de Setembro, 77 - $2^{\circ}$ 20551-030 - Rio de Janeiro, RJ

Recebido para publicação em 21/8/97

Aceito em 2/10/97
A dor precordial é sintoma freqüente na cardiomiopatia hipertrófica $(\mathrm{CMH})$, geralmente, se expressando no eletrocardiograma (ECG) pelo achado de isquemia miocárdica e apresentando relevante papel na fisiopatologia e na história natural desta doença ${ }^{1}$. Na maioria dos pacien- 
tes, o encontro de angina do peito na CMH é explicado por um dos seguintes mecanismos: redução da velocidade de fluxo e da reserva coronária ${ }^{2}$; existência de alterações nas pequenas artérias intramiocárdicas ${ }^{3}$; no tamanho inadequado das artérias coronárias em relação à massa hipertrofiada ${ }^{4}$; compressão dos ramos perfurantes septais da artéria descendente anterior (ADA) devido a hipertrofia septal ${ }^{5}$; pelo espasmo coronário ou decorrerente de processo aterosclerótico obstrutivo coronário ${ }^{6}$.

Gulotta $\mathrm{col}^{7}$, em 1972, foram os primeiros a referirem a associação entre a CMH e a doença arterial coronária (DAC) obstrutiva, sendo este percentual estimado entre 7,6 a 19\% dos pacientes com este tipo de cardiomiopatia ${ }^{8-12}$. No entanto, se separarmos os pacientes por faixa etária, veremos que nos $>45$ anos, ela se eleva para $25 \%^{8-12}$.

Em 1982, Sigwart e col ${ }^{13}$ relataram os efeitos agudos sobre as diversas regiões da parede ventricular e suas repercussões nas funções sistólica e diastólica, ocasionados pela oclusão coronária por cateter balão. Entretanto, somente mais tarde Gietzen e $\mathrm{col}^{14}$, em 1994, e Sigwart e col ${ }^{15}$, em 1995, observaram redução do gradiente da pressão intraventricular, nos casos de $\mathrm{CMH}$ obstrutiva, quando o cateter balão era expandido dentro do $1^{\circ}$ ramo septal da ADA. Este procedimento provoca infarto do miocárdio na zona do septo irrigada por aquele vaso, ocasionando fibrose e diminuição na massa miocárdica, com abolição ou redução da obstrução na via de saída do ventrículo esquerdo (VE).

Como este procedimento ainda é considerado controverso, decidimos analisar, entre os pacientes com CMH associado a DAC, aqueles que apresentassem envolvimento do 1/3 inicial da ADA, sua evolução e intercorrências, para melhor avaliarmos a resultante da necrose e da fibrose da região do septo, neste tipo de cardiomiopatia.

\begin{tabular}{|c|c|c|c|c|c|}
\hline \multicolumn{6}{|c|}{$\begin{array}{c}\text { Tabela I - Cardiomiopatia hipertrófica }(\mathrm{CMH}) \text { e doença arterial } \\
\text { coronária obstrutiva. Característica da população, tipo de acometimen- } \\
\text { to e tempo de diagnóstico da CMH }\end{array}$} \\
\hline $\mathrm{N}^{\circ}$ & Sexo & Idade & Cor & CMH tipo & Tempo diagnóstico \\
\hline 1 & M & 70 & B & obstrutivo & 182 meses \\
\hline 2 & M & 52 & B & obstrutivo & 78 meses \\
\hline 3 & $\mathrm{~F}$ & 67 & B & obstrutivo & 126 meses \\
\hline 4 & M & 65 & B & não obstrutivo & 180 meses \\
\hline 5 & M & 64 & B & não obstrutiva & 158 meses \\
\hline 6 & $\mathrm{~F}$ & 73 & B & obstrutivo & 122 meses \\
\hline
\end{tabular}

\section{Métodos}

Entre 158 pacientes com CMH vistos em nossa instituição, $7(4,43 \%)$ apresentavam DAC obstrutiva associada a esta cardiomiopatia, sendo que $6(3,79 \%)$ com comprometimento da ADA isolado ou associado a outras lesões coronárias, tendo sido estudados desde a definição do diagnóstico da CMH ou da cardiopatia isquêmica e acompanhados, visando correlacionar os achados da doença obstrutiva neste tipo de doença miocárdica. $\mathrm{O}$ delineamento do estudo foi observacional de casos prospectivos.

Os pacientes após o diagnóstico da lesão obstrutiva, evidenciada por meio da cinecoronariografia, foram estudados do ponto de vista epidemiológico, clínico e terapêutico, realizando exames complementares de rotina, ECG (trimestral), ecocardiograma (ECO) (anual), avaliação bioquímica (semestral), e exames complementares adicionais na dependência das alterações detectadas durante a evolução.

\begin{tabular}{|c|c|c|c|c|c|c|c|}
\hline $\mathrm{N}^{\circ}$ & Tipo & TempoCI & Fator Risco & Medicação & Dose & Cine $(\%)$ & Local \\
\hline 1 & AI & 76 meses & $\begin{array}{c}\mathrm{O}+\mathrm{HÁ}+\mathrm{T}+ \\
\mathrm{DM}+\mathrm{C}\end{array}$ & Propranolol & 240 & $\begin{array}{l}100 \mathrm{DA} \\
50 \mathrm{CD} \\
80 \mathrm{DP} \\
50 \mathrm{CIR}\end{array}$ & $\begin{array}{l}\text { após } 1^{\text {a }} \text { septal } \\
2 / 3 \\
1 / 3 \text { proximal } \\
2 / 3\end{array}$ \\
\hline 2 & IAM & 78 meses & $\mathrm{C}+\mathrm{S}+\mathrm{T}$ & Propranolol & 160 & $100 \mathrm{DA}$ & após $1^{\mathrm{a}}$ septal \\
\hline 3 & AI & 124 meses & $\mathrm{C}+\mathrm{O}$ & Propranolol & 240 & $\begin{array}{l}80 \mathrm{DA} \\
70 \mathrm{CD}\end{array}$ & $\begin{array}{l}\text { após } 1^{\mathrm{a}} \text { septal } \\
1 / 3 \text { proximal }\end{array}$ \\
\hline 4 & AI & 114 meses & $\begin{array}{c}\mathrm{C}+\mathrm{DM}+\mathrm{T}+ \\
\mathrm{DM}\end{array}$ & Verapamil & 240 & $\begin{array}{l}75 \mathrm{DA} \\
50 \mathrm{CIR}\end{array}$ & $\begin{array}{l}\text { após } 1^{\text {a }} \text { septal } \\
1 / 3 \text { distal }\end{array}$ \\
\hline 5 & AI & 104 meses & $\mathrm{C}+\mathrm{DM}+\mathrm{T}$ & Verapamil & 360 & $\begin{array}{l}60 \mathrm{DA} \\
70 \mathrm{DP} \\
60 \mathrm{CIR}\end{array}$ & $\begin{array}{l}\text { após } 1^{\text {a }} \text { septal } \\
1 / 3 \text { distal } \\
1 / 3 \text { proximal }\end{array}$ \\
\hline 6 & AI & 98 meses & $\mathrm{DM}+\mathrm{O}$ & Propranolol & 240 & $\begin{array}{l}80 \mathrm{DA} \\
50 \mathrm{CD}\end{array}$ & $\begin{array}{l}\text { após } 1^{\mathrm{a}} \text { septal } \\
1 / 3 \text { proximal }\end{array}$ \\
\hline
\end{tabular}

AI- angina instável; IAM- infarto agudo do miocárdio; CI- cardiopatia isquêmica; O- obesidade; DM- diabetes mellitus; T- tabagismo; C- hipercolesterolemia; HAhipertensão arterial; S- estresse; Cine- cinecoronariografia; DA- artéria descendente anterior; CIR- artéria circunflexa; CD- artéria coronária direita; DP- artéria descendente posterior. 


\section{Resultados}

Eram quatro homens e duas mulheres, todos da raça branca, com idades entre $52 \mathrm{e} 70(\mathrm{x}=65,16)$ anos, todos portadores da forma septal assimétrica da $\mathrm{CMH}$, quatro com o tipo obstrutivo e dois do não obstrutivo. O diagnóstico da $\mathrm{CMH}$ em cinco casos foi firmado antes do da cardiopatia isquêmica e, o outro, durante a caracterização da doença isquêmica. O tempo de diagnóstico da $\mathrm{CMH}$ variou de 78 a $182(\mathrm{x}=141)$ meses. (tab. I) Todos apresentavam dois ou mais fatores de risco para DAC, sendo a hipercolesterolemia o mais freqüente $(5 / 6-83,33 \%)$, seguida pelo diabetes mellitus e o tabagismo $(4 / 6-66,66 \%)$, a obesidade (3/6 $50 \%)$, o estresse (2/6 - 33,33\%) e a hipertensão arterial (HA) $(1 / 6-16,66 \%)$. A forma de apresentação da doença isquêmica obstrutiva foi, na maioria ( $5-83,33 \%$ casos), com angina instável e, em um, com infarto agudo do miocárdio (IAM), ocorrido entre o momento da caracterização da associação até $106(\mathrm{x}=42)$ meses após o diagnóstico da $\mathrm{CMH}$. A cinecoronariografia mostrou lesão obstrutiva de um vaso em um, de dois em três e de três ou mais em dois. A ADA estava comprometida entre 60 a 100\% (82,5\%) de sua luz, situando-se a obstrução em todos os casos após o $1^{\circ}$ ramo septal, as artérias coronária direita e circunflexa estavam acometidas em três casos com lesões entre 50 e $70 \%$, em dois casos observamos envolvimento da artéria descendente posterior (entre 70 - 80\%). Estes dados citados encontramse na tabela II.

O ECO, realizado na época do diagnóstico da $\mathrm{CMH}$, mostrou espessura do septo interventricular (SIV) entre 1,4 a 2,0 $(x=1,53) \mathrm{cm}$; a parede posterior do ventrículo esquerdo (ppVE) entre 0,8 a 1,2 (x=1,01)cm; o diâmetro diastólico do ventrículo esquerdo (DDVE) de 3,6 a 5,3 (x=4,55)cm; o diâmetro sistólico do ventrículo esquerdo (DSVE) de 2,0 a 3,7 $(\mathrm{x}=2,83) \mathrm{cm}$; o átrio esquerdo $(\mathrm{AE})$ de $2,9 \mathrm{a} 4,9(\mathrm{x}=4,13) \mathrm{cm}$; os quatro com a forma obstrutiva apresentavam o movimento sistólico anterior da valva mitral (MSAVM) e o gradiente sistólico estimado pelo Doppler entre o corpo e a via de saída do VEentre 28 a 120 ( $\mathrm{x}=56) \mathrm{mmHg}$, todos apresentando alterações no relaxamento diastólico e dois regurgitação mitral de grau leve (tab. III). No ECO, realizado antes da ocorrência de óbito ou no ano de 1996, observamos os seguintes valores: SIV de 1,1 a 1,7 ( $\mathrm{x}=1,4) \mathrm{cm}$; ppVE de 0,9 a 1,2 $(\mathrm{x}=1,03) \mathrm{cm}$;DDVEde4,0a5,8 $(\mathrm{x}=4,95) \mathrm{cm}$;DSVEde 2,6a3,9 $(\mathrm{x}=3,13) \mathrm{cm}$; AEde 3,5 a 5,1 $(\mathrm{x}=4,13) \mathrm{cm}$; gradiente sistólico de 10 a $20(x=15,75) \mathrm{mmHg}$, persistindo a alteração do relaxamento diastólico e a regurgitação mitral nos mesmos graus de antes da doença isquêmica obstrutiva (tab. III).

Os pacientes foram tratados para suas cardiopatias com nitrato sublingual para as crises de angina e com medicação inotrópica negativa e antisquêmica para a $\mathrm{CMH} \mathrm{e}$ para a cardiopatia isquêmica, tendo quatro recebido propranolole dois verapamil, com doses variando de 160 a $360 \mathrm{mg} /$ dia (tab. II).

A evolução mostrou que três pacientes aceitaram a indicação de cirurgia, sendo submetidos a revascularização miocárdica (RM), em um, associada a miomectomia septal; um outro foi submetido a angioplastia transluminal coronária e dois tratados clinicamente. Em todos, foi instituída terapia de correção dos fatores de risco cardiovascular, com medidas higiênicas, dietéticas e/ou químicas. Durante o tempo de acompanhamento, ocorreu 1 (16,66\%) óbito, em paciente submetido a cirurgia de RM que, apresentando angina do peito após 23 meses da cirurgia, foi novamente submetido a estudo hemodinâmico, que apresentava obstrução das pontes de safena, tendo sido reoperado 57 meses após a $1^{\mathrm{a}}$ intervenção, evoluindo com angina, a partir do $4^{\circ}$ mês do $2^{\circ}$ pós-operatório, tendo apresentado 35 meses após a última cirurgia, quadro de IAM vindo a falecer durante a internação em edema pulmonar agudo.

Um paciente, portador de cinco fatores de risco cardiovascular, teve controlados quatro deles, persistindo a obesidade, evoluiu com angina estável, aumento do AE $(4,4$ para $5,1 \mathrm{~cm})$, e episódios de fibrilação atrial paroxística, apresentando agravamento do quadro isquêmico durante os períodos de arritmia. $\mathrm{O}$ acréscimo da amiodarona ao tratamento medicamentoso reduziu o número de crises paroxísticas, permanecendo estável no momento (tab. IV).

Após a instalação do processo isquêmico septal e, por meio do ECO, observamos a redução da espessura do SIV, passando de 1,4 a 2,0 $(\mathrm{x}=1,53) \mathrm{cm}$ para $1,1 \mathrm{a} 1,7(\mathrm{x}=1,4) \mathrm{cm}$, sem alterar a espessura do ppVE (média 1,01 para $1,03 \mathrm{~cm}$ ), porém ocasionando discreto aumento no DDVE (média de 4,55 para $4,85 \mathrm{~cm}$ ) e no DSVE (média de 2,83 para $3,13 \mathrm{~cm}$ ), sem alterar a média do $\mathrm{AE}(4,13 \mathrm{~cm})$, ocorrendo diminuição na média do gradiente sistólico entre o corpo e a via de saída

\begin{tabular}{|c|c|c|c|c|c|c|c|c|c|c|c|c|c|c|c|c|}
\hline N. ${ }^{\circ}$ & \multicolumn{2}{|c|}{ SIV } & \multicolumn{2}{|c|}{ ppVE } & \multicolumn{2}{|c|}{ DDVE } & \multicolumn{2}{|c|}{ DSVE } & \multicolumn{2}{|c|}{$\mathrm{AE}$} & \multicolumn{2}{|c|}{ MSAVM } & Gradiente & \multicolumn{2}{|c|}{ Relaxam. } & $\mathrm{IM}$ \\
\hline 1 & 2,0 & 1,7 & 1,0 & 1,0 & 5,3 & 5,6 & 2,8 & 3,4 & 4,4 & 5,1 & + & + & $28 \quad 10$ & $<$ & $<$ & $-\quad-$ \\
\hline 2 & 1,4 & 1,2 & 0,9 & 1,0 & 4,5 & 4,8 & 3,1 & 2,7 & 4,3 & 3,5 & + & + & 12020 & $<$ & $<$ & L L \\
\hline 3 & 1,4 & 1,1 & 0,8 & 1,0 & 3,6 & 4,6 & 2,2 & 2,6 & 2,9 & 2.9 & + & + & $34 \quad 15$ & $<$ & $<$ & $-\quad-$ \\
\hline 4 & 1,8 & 1,5 & 1,0 & 0,9 & 5,3 & 5,8 & 3,7 & 3,9 & 4,0 & 4,2 & - & - & $\begin{array}{ll}0 & 0\end{array}$ & $<$ & $<$ & - - \\
\hline 5 & 1,6 & 1,4 & 1,2 & 1,2 & 3,8 & 4,0 & 2,0 & 2,6 & 4,9 & 4,5 & - & - & 0 & $<$ & $<$ & $-\quad-$ \\
\hline 6 & 1,8 & 1,5 & 1,2 & 1,1 & 4,8 & 4,9 & 3,2 & 3,6 & 4,3 & 4,6 & + & + & $42 \quad 18$ & $<$ & $<$ & L L \\
\hline
\end{tabular}




\begin{tabular}{|c|c|}
\hline \multicolumn{2}{|r|}{$\begin{array}{l}\text { Tabela IV - Evolução dos pacientes com a associação da cardiomiopatia } \\
\text { hipertrófica e cardiopatia isquêmica obstrutiva da artéria } \\
\text { descendente anterior }\end{array}$} \\
\hline $\mathrm{N}^{\circ}$ & Evolução \\
\hline 1 & $\begin{array}{l}\text { Não aceitou cirurgia, controlados } \mathrm{T}+\mathrm{DM}+\mathrm{HA}+\mathrm{C} \text {, persiste obesidade, } \\
\text { angina estável, aumento do átrio esquerdo com episódios de fibrilação } \\
\text { atrial paroxística, agravamento da angina nos períodos de FA, acres- } \\
\text { centado amiodarona, estável. }\end{array}$ \\
\hline 2 & $\begin{array}{l}\text { Submetido à angioplastia coronária, controlado } \mathrm{C}+\mathrm{T} \text {, angina estável, } \\
\text { bem. }\end{array}$ \\
\hline 3 & $\begin{array}{l}\text { Cirurgia de revascularização miocárdica + miomectomia há } 104 \text { meses } \\
\text { controlado } \mathrm{O}+\mathrm{C} \text {, atualmente bem. }\end{array}$ \\
\hline 4 & $\begin{array}{l}\text { Cirurgia de revascularização miocárdica há } 84 \text { meses, controlado o } \\
\mathrm{DM}+\mathrm{C} \text {, angina estável, bem. }\end{array}$ \\
\hline 5 & $\begin{array}{l}\text { Cirurgia de revascularização miocárdica há } 92 \text { meses, evoluiu com an- } \\
\text { gina, reoperado } 57 \text { meses após, óbito } 35 \text { meses depois por IAM. }\end{array}$ \\
\hline & Não aceitou cirurgia, angina estável, bem. \\
\hline \multicolumn{2}{|r|}{$\begin{array}{l}\text { T- tabagismo; DM- diabetes mellitus; HA- hipertensão arterial; } \\
\text { hipercolesterolemia; O- obesidade; IAM- infarto agudo do miocárdio; FA- } \\
\text { fibrilação atrial. }\end{array}$} \\
\hline
\end{tabular}

do VE de 56 para $15,75 \mathrm{mmHg}$. Alterações que acreditamos não tenham sido devidas somente a instalação da isquemia e conseqüente fibrose, mas também decorrentes da ação medicamentosa de agentes inotrópicos, utilizados pelos pacientes como o propranolol e o verapamil.

\section{Discussão}

Nossa população de CMH é constituída, em sua maioria, por portadores da forma septal assimétrica $(86,6 \%)$, seguida pelas formas apical $(8,34 \%)$, concêntrica $(2,53 \%)$, medioventricular e lateral (1,26\% cada). Tivemos $7(4,43 \%)$ casos da associação com a cardiopatia isquêmica obstrutiva, sendo em um restrita ao comprometimento único da artéria descendente posterior e os restantes com acometimento que sempre incluía a ADA $(3,79 \%)$.

Com a finalidade de avaliar o risco de desenvolvimento de DAC em jovens ${ }^{16}$, estudo realizado entre 4111 pacientes de 23 a 35 anos, moradores de centros urbanos, mostrou, através do ECO, que a CMH foi observada em $7(0,17 \%)$ desses pacientes, sendo sua prevalência de $0,26 \%$ nos homens e $0,09 \%$ nas mulheres, mais freqüente entre negros $(0,24 \%)$ do que brancos $(0,10 \%)$, e encontrada em cerca de dois entre 1000 adultos jovens.

São raros os casos onde a DAC encontrada em pacientes com CMH decorre de anomalias congênitas desses vasos, sendo relatados três casos por Walston e Behar ${ }^{8}$ e um por Lardani e $\operatorname{col}^{9}$. Em nossa casuística, não encontramos esta associação e nem entre todos os pacientes com $\mathrm{CMH}$, vistos em nossa instituição, que realizaram CINE.

O diagnóstico clínico da associação entre a DAC e a CMH é difícil de ser firmado, pois as doenças apresentam sintomas e sinais idênticos (angina, bulha adicional $\left(4^{\mathrm{a}}\right)$ e sopros), necessitando atenção redobrada, principalmente, quando dois ou mais fatores de risco coronário estão presentes e quando o propranolol não é capaz de abolir ou con- trolar os sintomas. Bensaid e col ${ }^{17}$ observaram que, em apenas $50 \%$ da população analisada com esta associação, o propranolol foi eficaz em reduzir ou abolir a angina do peito.

A angina do peito é freqüente na hipertrofia ventricular esquerda (HVE), principalmente entre aqueles com $\mathrm{CMH}$, associada a fibrose devido a isquemia miocárdica intermitente ${ }^{1,4,12}$. Nos últimos anos, vários trabalhos demostraram o papel reversível desta isquemia na gênese destas alterações, através da cintigrafia miocárdica com tálio 201, podendo decorrer da obstrução coronária aterosclerótica ou de mecanismos que aumentam a necessidade do fluxo coronário regional ou impedem a reserva do fluxo hiperêmico máximo ${ }^{18-20}$.

As lesões coronárias obstrutivas $<60 \%$, consideradas como de menor significado nos pacientes sem hipertrofia ventricular, ganham maior relevo nos portadores de $\mathrm{CMH}$, contribuindo para o aparecimento da angina do peito e da isquemia miocárdica ${ }^{8}$. Assim, consideramos, como representativo em nossa série, as lesões $\geq 60 \%$ na ADA, tendo sido observado em apenas um paciente esta dimensão, nos demais eram $>75 \%$ e em três eram completas $(100 \%)$. Em todos os casos esta lesão ocorria após a emergência do $1^{\circ}$ ramo septal da ADA, sendo de se esperar uma grande extensão na área do comprometimento da massa muscular septal, desencadeando alteração da função ventricular por modificação na nutrição coronária.

Kimball e $\mathrm{Col}^{4}$, estudando o diâmetro da ADA e o relacionando com doenças que aumentam a massa ventricular, constataram que ele é de $3,2 \pm 0,54 \mathrm{~mm}$ em indivíduos normais, de 3,82 $\pm 0,71 \mathrm{~mm}$ nos com estenose valvar aórtica e de $4,72 \pm 0,81 \mathrm{~mm}$ nos com CMH ( $\mathrm{p}<0,05)$, isto é, apresentam maior diâmetro os pacientes em condições de maior hipertrofia. Maron e col ${ }^{21}$ observaram, no entanto, aumento do espessamento da média e da íntima das artérias coronárias, secundárias a proliferação da musculatura lisa, por aumento no teor de colágeno, reduzindo o lúmem dos vasos. Estes achados foram confirmados por Tanaka e $\mathrm{col}^{3}$ que os relacionaram com os graus de hipertrofia miocítica regional, de desarranjo das fibras cardíacas e de fibrose miocárdica localizada.

Analisando a velocidade de fluxo na ADA, utilizando a ecocardiografia transesofágica com Doppler pulsado, Tomochika e $\operatorname{col}^{22}$ observaram existir diferença entre os portadores de $\mathrm{CMH}$ não obstrutiva e os normais, sendo a velocidade de pico do fluxo sistólico menor nos que apresentavam CMH $(34 \pm 11 \mathrm{~cm} / \mathrm{s}$ vs $7 \pm 30 \mathrm{~cm} / \mathrm{s}$, com $\mathrm{p}<0,005)$, com correlação inversa entre o pico do fluxo sistólico e a espessura do SIV ( $r=0,81 ; p<0,01)$. Nenhuma diferença significativa foi observada na velocidade de pico do fluxo diastólico, entretanto, foram observadas alterações significativas na CMH no início da diástole, com o prolongamento do tempo de aceleração da velocidade de fluxo na ADA $(210 \pm 67$ vs $95 \pm 15 \mathrm{~ms}, \mathrm{p}<0,01)$ e a redução da aceleração da freqüência $(3,6 \pm 2,0$ vs $6,6 \pm 1,8 \mathrm{~m} / \mathrm{s} 2, \mathrm{p}<0,02)$. Atribuíram os achados sistólicos, ao aumento da pressão perivascular no SIV espessado, e relacionaram a alteração do relaxamento ventricular, às modificações vistas no início e no meio da diástole ${ }^{22}$. 
A CMH obstrutiva apresenta mortalidade anual de $4 \%{ }^{23-25}$ e mortalidade pós miectomia $<2 \% / a^{23,26,27}$. A presença de DAC representa risco adicional na mortalidade na $\mathrm{CMH}$, sendo considerada de $10 \%$ ao ano em comparação aos pacientes sem esta complicação que é de 2,2\% ao ano ${ }^{11,25,28}$.

Entre os portadores deste tipo de cardiomiopatia, os pacientes com esta associação são mais velhos e apresentam maior incidência de extra-sistolia ventricular, aumento do DDVE $>5,5 \mathrm{~cm}$, com diminuição do gradiente de pressão intraventricular do VE e menor número de casos onde o MSAVM é encontrado ${ }^{8-10,29,30}$. A nossa casuística era composta por pacientes $>52$ anos, com idade média de 65,16 anos, todos caucasianos, com DDVE, na ocasião do diagnóstico, sempre com valores $<5,5 \mathrm{~cm}$, estando o MSAVM presente nos quatro com a forma obstrutiva, apresentando gradiente de pressão intraventricular entre $28-120 \mathrm{mmHg}$ (média de $56 \mathrm{mmHg}$ ), porém, três dos quatro com valores $<42 \mathrm{mmHg}$. Nossa série tem características diferentes das outras descritas, refletindo talvez a heterogeneidade entre os pacientes avaliados.

Quanto a faixa etária, todos estavam entre a $6^{\mathrm{a}}$ e a $8^{\mathrm{a}}$ décadas (52 a 70 anos, com média de 65,16) e superior às séries descritas, que oscilavam entre 44 anos ${ }^{11}$ a média de 58 anos $^{7,8,30,31}$, porém próxima a de 64 anos referida por Cokkinos e $\mathrm{col}^{10}$. O mais jovem paciente descrito na literatura era um homem de 35 anos ${ }^{10}$. Os casos que vimos eram de pacientes com mais idade, devendo ser realçado serem portadores de dois ou mais fatores de risco cardiovascular.

A espessura do SIV variava de 1,4 a 2,0cm, com média de $1,53 \mathrm{~cm}$, próxima a observada por Lazzeroni e col ${ }^{11} \mathrm{de}$ $1,6 \pm 0,3 \mathrm{~cm}$, odoDDVEde $3,6 \mathrm{a} 5,3 \mathrm{~cm}(\mathrm{x}=4,55)$, inferior ao referido no estudo, que foi em $45 \%$ da série $>5,5 \mathrm{~cm}$, realçando ser essa população mais jovem do que a nossa, refletindo a ação da DAC mais precoce em miocárdio hipertrofiado e com maior necessidade de oxigênio.

Em estudo que tenta definir o significado da DAC na CMH, Lazzeroni e col ${ }^{11}$ observaram que os componentes da associação apresentavam menor espessura do SIV $(1,6 \pm 0,3 \mathrm{~cm})$ quando comparados aos que não tinham DAC $(2,1 \pm 0,6 \mathrm{~cm})$, além de terem encontrado, mais freqüentemente, DDVE $>5,5 \mathrm{~cm}$ nos pacientes com a associação (45\%) do que nos que tinham somente $\mathrm{CMH} \mathrm{(7 \% ),} \mathrm{consta-}$ tando que os pacientes que apresentaram IAM, mostraram maior DDVE $(6,2 \mathrm{~cm})$ e menor fração de ejeção $(38,6 \%)^{11}$.

$\mathrm{O}$ achado de dois ou mais fatores de risco cardiovascular foi o marcador de maior relevância para a suspeição da DAC, devendo ser mencionado que a hipercolesterolemia esteve presente em $83,33 \%$ da série, e que o diabetes mellitus e o tabagismo foram encontrados em $66,66 \%$, destacando-se o papel desses fatores na resposta endotelial coronária, em pacientes com lesão obstrutiva e hipertrofia miocárdica, onde maior consumo de oxigênio é esperado por grama de miocárdio. O encontro da HVE no ECG foi visto em cinco dos $6(83,33 \%)$ pacientes, sendo em apenas um relacionado a associação com a HA (de grau leve) e nos outros decorrentes da associação da CMH e da DAC.
Foi observada na raça negra maior mortalidade entre os homens, principalmente naqueles com $\operatorname{HVE}(5,4 / 100$ pacientes-ano) em relação aos sem $\operatorname{HVE}$ (2,58/100 pacientes) e em comparação às mulheres $(3,21 / 100$ pacientes-ano nas com HVE e 0,66/100 pacientes-ano nas sem HVE) acompanhadas por cinco anos, realçando-se neste estudo a utilização da cinecoronariografia a fim de afastar o acometimento simultâneo da aterosclerose coronária, demostrando o papel isolado da HVE no prognóstico da $\mathrm{CMH}^{32}$.

Após o trabalho de Gullota e col ${ }^{7}$, vários autores descreveram o encontro de lesões arteriais obstrutivas na circulação coronária. Epstein e col ${ }^{33}$, analisando 20 pacientes com CMH, encontraram sete que apresentavam obstrução $>50 \%$. Marcus e col ${ }^{34}$ descreveram caso de infarto subendocárdico em paciente de 55 anos com CMH e que apresentava lesão obstrutiva isolada da coronária direita, tratado com miomectomia e revascularização coronária, desaparecendo a síndrome anginosa. No entanto, no mesmo período foram publicados vários relatos demostrando a associação entre $\mathrm{CMH}$, ECG com sinais de infarto e coronariografia revelando artérias normais ${ }^{35-37}$.

Na maioria dos casos descritos da associação da CMH e DAC, observamos acometimento dos três ramos principais coronários, demonstrando a gravidade do comprometimento aterosclerótico no desencadeamento da lesão obstrutiva $^{7-11,29-31}$. Nesses trabalhos não são mencionadas as freqüências da ocorrência dos fatores de risco cardiovascular. No nosso estudo, encontramos, em apenas um caso, lesão única da ADA, nos demais, lesões em dois ramos em dois, e nos restantes, de três, de acordo com a literatura ${ }^{7-11,29-31}$.

A ADA freqüentemente é a de maior freqüência de comprometimento, sendo $>70 \%$ na maioria das séries relacionadas ${ }^{8-10,29}$ e foi a lesão de referência no nosso estudo.

Entre 28 pacientes com a associação da $\mathrm{CMH}$ e da DAC, submetidos a revascularização cirúrgica do miocárdio por Siegman e col ${ }^{31}$, foram observados 5 (18\%) óbitos, quatro imediatos (dois por baixo débito cardíaco, um por comunicação interventricular (CIV) iatrogênica e um por mediastinite) e um no $2^{\circ}$ mês pós-operatório, além de três outros não diretamente relacionados à cirurgia. Entre os 20 sobreviventes, em período de acompanhamento, variando de quatro meses a 10,8 anos (média de 4,8 anos), 19 melhoraram dos seus sintomas. Foi observada nos pacientes submetidos a miotomia/miectomia, a presença de CIV, complicação iatrogênica acometendo cinco dos 24 (21\%) casos, freqüente naqueles que apresentavam espessura do SIV $<2,0 \mathrm{~cm}$, sendo que dois deles vieram a falecer em decorrência desta complicação ${ }^{31}$.

Avaliando as séries de tratamento cirúrgico, observamos na de Gill e col ${ }^{30}$ que dos 21 operados, sendo sete com revascularização cirúrgica, um faleceu e outro permaneceu com sintomas que motivaram a intervenção, e os 14 restantes foram submetidos a revascularização acompanhada por miotomia, tendo todos alívio dos sintomas. No entanto, Stewart e Schreiner ${ }^{29}$, entre os cinco pacientes submetidos a revascularização e miotomia, tiveram $40 \%$ de óbitos no 
pós-operatório, em comparação a outros 14 pacientes submetidos a miotomia isolada, sem nenhum óbito.

$\mathrm{Na}$ nossa série, dos três submetidos a revascularização cirúrgica, um também foi submetido a miectomia, sem terem sido observadas complicações na fase precoce. Um paciente submetido apenas a RM, ocluiu as anastomoses venosas, apresentando exacerbação da angina (caso $\mathrm{n}^{\circ} 5$ ), com seus fatores de risco controlados, tendo sido reoperado, evoluindo 35 meses após esta $2^{\mathrm{a}}$ cirurgia de revascularização para IAM, e vindo a falecer, em decorrência de extensa área de lesão miocárdica por edema pulmonar agudo, 92 meses após a $1^{\text {a }}$ revascularização.

Tivemos um caso onde foi realizada a angioplastia transluminal coronária, com sucesso, conseguindo-se transpor lesão total e dilatar lesão única da ADA, ficando o paciente assintomático em uso de propranolol, com redução do gradiente de 120 para $20 \mathrm{mmHg}$, com diminuição da espessura do SIV de 1,4 para 1,2cm e discreto aumento do DDVE de 4,5 para $4,8 \mathrm{~cm}$, sem apresentar novo quadro isquêmico.

Lazzeroni e col ${ }^{11}$ observaram aumento da mortalidade no grupo com a associação (36\%) quando comparado com a CMH isolada ( $8 \%$ ) com significância estatística de 0,05 , em seguimento de 3,6 anos (4/11 vs 7/85, e mortalidade de $10 \%$ ao ano vs 2,2\% ao ano). Em nossa população tivemos o índice de mortalidade de $16,66 \%$, inferior ao descrito por Lazzeroni e col ${ }^{11}$, em avaliação média de 141 meses.

A evolução da CMH para a forma dilatada, especialmente quando o quadro anginoso estiver presente, poderá ser altamente duvidosa da associação da DAC, sendo necessário a realização de estudo cinecoronariográfico para afastar tal acometimento ${ }^{11}$. Na nossa população, não fizemos uso desta suspeita na avaliação da $\mathrm{CMH}$, uma vez que não presenciamos esta evolução para a dilatação, como forma de exteriorização da associação.

Entre os 16/85 pacientes (19\%) vistos por Cokkinos e col ${ }^{10}$ com DAC fixa e $>60 \%$ em um ou mais vasos, cinco apresentavam lesão da ADA $>90 \%$ e quatro entre 75 a $89 \%$, sem apresentarem nenhuma evidência de circulação colateral, justificado pelo miocárdio hipertrofiado poder interferir com a formação dos vasos colaterais. A angina de peito esteve presente em 13 dos 16 pacientes, sendo que em cinco notada exacerbação do quadro anginoso ${ }^{10}$. Nesse grupo, cinco foram submetidos a tratamento cirúrgico, com melhora da síndrome anginosa, sendo que um caso evoluiu para óbito três anos após por insuficiência cardíaca congestiva.

Os trabalhos empregando a oclusão do $1^{\circ}$ ramo septal da ADA têm demonstrado a redução ou abolição do gradiente de pressão nos casos de CMH obstrutiva, tendo a técnica sido acrescida da instilação de álcool puro, provocan- do necrose do SIV irrigado por esse vaso que, posteriormente, é substituído por fibrose, fazendo diminuir ou abolir o gradiente intraventricular ${ }^{13-15}$. Os nossos pacientes apresentavam lesão da ADA, após a emergência do $1^{\circ}$ ramo septal, logo apresentavam comprometimento da irrigação septal, acometendo as suas porções média e inferior, contribuindo para o remodelamento da cavidade do VE. Todos os nossos pacientes com a forma obstrutiva foram medicados com propranolol que, em conjunto com a fibrose do septo, provocada pela doença isquêmica, foram responsáveis pela redução dos gradientes de pressão intraventricular. Wigle e col ${ }^{38}$ supõem que os bloqueadores beta-adrenérgicos seriam melhores indicados na $\mathrm{CMH}$ obstrutiva na forma latente, e na de repouso com obstrução leve a moderada, acreditando serem menos eficazes nas obstruções graves, embora outros autores tenham referido bons resultados nos casos com grande obstrução ${ }^{39,40}$.

Tivemos um caso que evoluiu com aumento significativo do AE de 4,4 para $5,1 \mathrm{~cm}$, tendo o paciente apresentado episódios de fibrilação atrial paroxística, com agravamento do quadro anginoso nos períodos que cursavam com a arritmia. Já vínhamos observando que pacientes com $\mathrm{CMH}$, apresentando aumento do $\mathrm{AE}>4,7 \mathrm{~cm}$, possuem maior probabilidade de fibrilar, principalmente com a forma septal assimétrica e após a faixa etária do $5^{\circ}$ decênio, além de expostos a maior risco de tromboembolismo e de disfunção miocárdica $^{41}$

A cardiopatia isquêmica obstrutiva é uma complicação rara na CMH, ocorrida em nossa casuística em 3,79\%, acometendo pacientes mais velhos, com idade média de 65 anos e surgindo em portadores de dois ou mais fatores de risco cardiovascular. Apesar da doença comprometer a irrigação do septo, com necrose e fibrose, esta não agravou a CMH. Os pacientes tiveram boa evolução clínica, permanecendo em acompanhamento médio de 78 a 182 (141) meses, após a caracterização da CMH, observou-se um $(16,66 \%)$ óbito, um caso evoluiu com fibrilação atrial com exacerbação do quadro anginoso e quatro estão compensados com drogas inotrópicas negativas (melhorando a disfunção diastólica) e com atividade antiisquêmica. Acreditamos que a lesão da ADA não cause efeito maléfico nos pacientes com CMH e, talvez, a boa evolução observada possa ser a que vem sendo encontrada nos pacientes submetidos a angioplastia transluminal do $1^{\circ}$ ramo septal da ADA, com a instilação de álcool nessa região, ocasionando necrose e fibrose do septo.

A DAC, devido ao envolvimento da ADA, é bem tolerada nos pacientes com $\mathrm{CMH}$ septal assimétrica, participando do processo fibrótico septal, não interferindo com o desempenho ventricular e não representando problema adverso na evolução da CMH. 


\section{Referências}

1. Wigle ED, Sasson Z, Henderson MA et al - Hypertrophic cardiomyopathy: the importance of the site and the extent of hypertrophic - a review. Prog Cardiovasc Dis $1985 ; 28: 1-83$.

2. Memmola C, Iliceto S, Napoli VF, Cavallari D, Santoro G, Rizzon P-Coronary flow dynamics and reserve assessed by transesophageal echocardiography in obstructive hypertrophic cardiomyopathy. Am J Cardiol 1994; 74: 1147-51.

3. Tanaka M, Fukiwara H, Onodera T et al - Quantitative analysis of narrowings of intramyocardial small arteries in normal hearts, hypertensive hearts and hearts with hypertrophic cardiomyopathy. Circulation 1987; 75: 1130-9.

4. Kimball BP, LiPreti V, Bui S, Wigle ED - Comparison of proximal left anterior descending and circunflex coronary artery dimensions in aortic valve stenosis and hypertrophic cardiomyopathy. Am J Cardiol 1990; 65: 767-71.

5. Pichard AD, Meller J, TeichholzLE, Lipnik S, Gorlin R, Herman MV - Septal perforator compression (narrowing) in idiopathic hypertrophic subaortic stenosis. Am J Cardiol 1977; 40: 310-14.

6. Nishimura K, Nosaka H, Saito T, Nobuyoshi M- Another possible mechanism of angina in hypertrophic cardiomyopathy. Circulation 1983; 68(suppl. III): 162.

7. Gulotta SJ, Hamby RI, Aronson AL, Ewing K - Coexistent idiopathic hypertrophic subaortic stenosis and coronary arterial disease. Circulation 1972; 46: 890-6.

8. Walston A II, Behar VS - Spectrum of coronary artery disease in idiopathic subaortic stenosis. Am J Cardiol 1976; 38: 12-6.

9. Lardani H, Serrano JA, Villami RJ - Hemodynamics and coronary angiography in idiopathic hypertrophic subaortic stenosis. Am J Cardiol 1978; 41: 476-81.

10. Cokkinos DV, KrajcerZ, Leachman RD-Coronary artery disease in hypertrophic cardiomyopathy. Am J Cardiol 1985; 55: 1437-8.

11. Lazzeroni E, Rolli A, Aurier E, Botti G-Clinical significance of coronary artery disease in hypertrophic cardiomyopathy. Am J Cardiol 1992; 70: 499-501.

12. Harjai HJ, Cheirif J, Murgo JP - Ischemia and atherosclerotic coronary artery disease in patients with hypertrophic cardiomyopathy : a review of incidence, pathophysiological mechanisms, clinical implications and management strategies. Coronary Artery Dis 1996; 7: 183-7.

13. Sigwart U, Grbic M, Essinger A, River JL - L'effet aigu d'une occlusion coronarienne par ballonet de la dilatation transluminale. Schweiz Med Wschr 1982; 45: 1631.

14. Gietzen F, Leuner C, Gerenkamp T, Kuhn H - Relief of obstruction in hypertrophic cardiomyopathy by transient occlusion of the first septal branch of the left coronary artery. Eur Heart J 1994; 15: 125.

15. Sigwart U-Nonsurgical myocardial reduction: a new treatment for hypertrophic obstructive cardiomyopathy. Lancet 1995; 346: 221.

16. Maron BJ, Gardin JM, Flack JM, Gidding SS, Kurosaki TT, Bild DE-Prevalence of hypertrophic cardiomyopathy in a general population of young adults echocardiographic analysis of 4111 subjects in the CARDIA study. Circulation 1995; 92: 785-9.

17. Bensaid $\mathbf{J}$ - Idiopathic hypertrophic subaortic stenosis and associated coronary artery disease. Angiology 1979; 58: 585-93.

18. Hanrath P, Mathey D, Montz R et al - Myocardial thallium-201 imaging in hypertrophic obstructive cardiomyopathy. Eur Heart J 1981; 2: 177-85.

19. O'Gara PT, Bonow RO, Maron BJ et al - Myocardial perfusion abnormalities in patients with hypertrophic cardiomyopathy: assessment with thallium-201 emission computed tomography. Circulation 1987; 76:1214-23.

20. Pitcher D, Wainwright R, Mansey M, Curry P, Lowton F - Assessment of chest pain in hypertrophic cardiomyopathy using exercise thallium-201 myocardial imaging scintigraphy. Br Heart J 1989; 44: 650-6.
21. Maron BJ, Wolfson JK, Epstein SE, Roberts WC - Intramural ("small vessel”) coronary artery disease in hypertrophic cardiomyopathy J Am Coll Cardiol 1986; 8: 545-57.

22. Tomochika Y, Tanaka N, Wasaki Y et al-Assessment of flow profile of left anterior descending coronary artery in hypertrophic cardiomyopathy by transesophageal pulsed Doppler echocardiography. Am J Cardiol 1993; 72: 1425-30.

23. Swan DA, Bell B, Oakley C, Goodwin JF - Analysis of symptomatic course and prognosis and treatment of hypertrophic obstructive cardiomyopathy. Br Heart J 1971; 33: 671-85.

24. Adelman AG, Wigle ED, Ranganathan $\mathrm{N}$ et al - The clinical course in muscular subaortic stenosis : a retrospective and prospective study of 60 hemodynamically proved cases. Ann Intern Med 1972; 77: 515-25

25. Shah PM, Adelman AG, Wigle ED et al - The natural (and unnatural ) history of hypertrophic obstructive cardiomyopathy. Circ Res 1974; 35(suppl. II): 179-95.

26. Maron BJ, Epstein SE, Morrow AG - Symptomatic status and prognosis of patients after operation for hypertrophic obstructive cardiomyopathy : efficacy of ventricular septal myotomy and myectomy. Eur Heart J 1983; 4(suppl F): 175 85.

27. tem Berg JM, Suttorp MJ, Knaepen PJ, Ernest SMPG, Vermeulen FEE, Jaarsma W - Hypertrophic obstructive cardiomyopathy: initial results and long-term follow-up after Morrow septal myectomy. Circulation 1994; 90: 1781-5.

28. Hardarson T, De la Calzada CS, Curiel R, Goodwin JF- Prognosis and mortality of hypertrophic obstructive cardiomyopathy. Lancet 1973; 1: 732-9.

29. Stewart S, Schreiner B - Coexisting idiopathic hypertrophic subaortic stenosis and coronary artery disease. J Thorac Cardiovasc Surg 1981; 82: 278-80.

30. Gill CG, Duda AM, Kitazume H, Kramer JR, Loop FD - Idiopathic hypertrophic subaortic stenosis and coronary atherosclerosis. J Thorac Cardiovasc Surg 1982; 84: 856-60.

31. Siegman I, Maron BJ, Permut L, McIntosh CL, Clark RE-Results of operation for coexistent obstructive hypertrophic cardiomyopathy and coronary artery disease. J Am Coll Cardiol 1989; 13: 1527-33.

32. Liao Y, Cooper RS, Mensah GA, McGee DL - Left ventricular hypertrophy has a greater impact on survival in women than in men. Circulation 1995; 92: 805-10.

33. Epstein SE, Henry WL, Clark CE et al - Asymmetric septal hypertrophy. Ann Inter Med 1974; 81: 650-80.

34. Marcus GB, Popp RL, Stinson ED - Coronary artery disease with idiopathic hypertrophic subaortic stenosis. Lancet 1974; 1: 901-3.

35. Stein PD, Solis RM, Broks HLet al - Vectocardiogram simulating myocardial infarction in idiopathic hypertrophic subaortic stenosis. Dis Chest 1968; 54: 469-74.

36. Welch CC, Scott LP III - Muscular subaortic stenosis masquerading as myocardial infarction. Am J Med Sci 1965; 249: 8-12.

37. Prescott R, Quinn JS, Littmann D - Electrocardiographic changes in hypertrophic subaortic stenosis which simulate myocardial infarction. Am Heart J 1963; 66: 42-8

38. Wigle ED, Rakowski H, Kimball BP, Williams W - Hypertrophic cardiomyopathy - clinical spectrum and treatment. Circulation 1995; 92: 1680-92.

39. Stenson RE, Flamm Jr MD, Harrinson DC, Hancock EW - Hypertrophic subaortic stenosis: clinical and hemodynamic effects of long-term propranolol therapy. Am J Cardiol 1973; 31: 763-73.

40. Frank MJ, Abdulla AM, Canedo MI, Saylors RE-Long-term medical management of hypertrophic obstructive cardiomyopathy. Am J Cardiol 1978; 42: 993-1001.

41. Albanesi $F^{\circ}$ FM, Girardi JM, Castier MB, GinefraP-Influência da fibrilação atrial na história natural da cardiomiopatia hipertrófica. Arq Bras Cardiol 1994; 62 337-41. 